\title{
KINEMATIC MAPPING APPLICATION TO APPROXIMATE TYPE AND DIMENSION SYNTHESIS OF PLANAR MECHANISMS
}

\author{
M. John D. Hayes, Tim Luu \\ Department of Mechanical and Aerospace Engineering, Carleton University \\ 1125 Colonel By Drive, Ottawa, ON, K1S 5B6, Canada \\ jhayes@mae.carleton.ca, tim_luu@hotmail.com \\ Xiao-Wen Chang \\ School of Computer Science, McGill University \\ 3480 University Street, Montréal, QC, H3A 2A\%, Canada \\ chang@cs.mcgill.ca
}

\begin{abstract}
Kinematic mapping is used for preliminary development of an algorithm for the approximate synthesis of planar four-bar mechanisms for rigid body guidance. Both dyad type and dimensions are determined. Planar mechanism coupler motions are represented as the curves of intersection of a pair of quadric constraint surfaces, one for each of two dyads. The problem reduces to identifying the two best constraint surfaces in the pencil of quadrics containing the curve. The overdetermined synthesis equations are linear in the unknown surface shape coefficients, and their products. Non-trivial solutions exist only in the nullspace of the coefficient matrix. While the algorithm remains incomplete, results presented herein are encouraging.
\end{abstract}

Keywords: Approximate type and dimensional synthesis, rigid body guidance, kinematic mapping, singular value decomposition.

\section{Introduction}

The kinematic synthesis of robot mechanical systems has been the focus of a significant volume of research; in particular, robots whose mechanical systems are parallel kinematic chains. For example Shirkhodaie and Soni, 1987 use a reasonably straightforward Cartesian approach for kinematic synthesis of planar parallel robots with three degrees-offreedom (DOF). Murray and Pierrot, 1998 present an algebraic algorithm, based on quaternions, for synthesis of planar three-legged platforms given $n$-positions. We intend to develop an $n$-pose synthesis algorithm for both dyad type and dimensions based on the geometry of the kinematic image of the desired coupler positions and orientations. But, 
as with many things kinematic, while the proposed concept is elegantly simple, the devil is in the details.

\section{Kinematic Mapping}

Kinematic mapping was introduced independently by Blaschke, 1911, and Grünwald, 1911. One can consider the relative displacement of two rigidbodies in the plane as the displacement of a Cartesian reference coordinate frame $E$ attached to one of the bodies with respect to a Cartesian reference coordinate frame $\Sigma$ attached to the other. Without loss of generality, $\Sigma$ may be considered fixed with $E$ free to move, as is the case with the fourbar mechanism illustrated by Figure 1 .

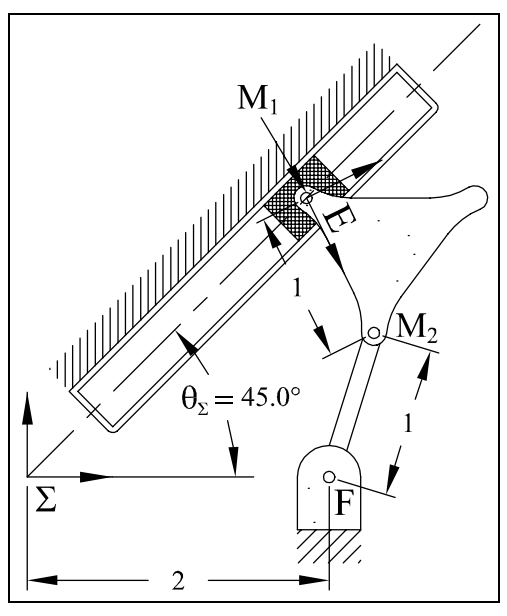

Figure 1. Planar $R R R P$ linkage.

The homogeneous coordinates of points represented in $E$ are given by the ratios $(x: y: z)$. Those of the same points represented in $\Sigma$ are given by the ratios $(X: Y: Z)$. The position of a point $(X: Y: Z)$ in $E$ in terms of the basis of $\Sigma$ can be expressed compactly as

$$
\left[\begin{array}{l}
X \\
Y \\
Z
\end{array}\right]=\left[\begin{array}{ccc}
\cos \varphi & -\sin \varphi & a \\
\sin \varphi & \cos \varphi & b \\
0 & 0 & 1
\end{array}\right]\left[\begin{array}{l}
x \\
y \\
z
\end{array}\right],
$$

where the pair $(a, b)$ are the $(X / Z, Y / Z)$ Cartesian coordinates of the origin of $E$ expressed in $\Sigma$, and $\varphi$ is the orientation of $E$ relative to $\Sigma$, respectively.

The essential idea of kinematic mapping is to map the three homogeneous coordinates of the pole of a planar displacement, in terms of $(a, b, \varphi)$, to the points of a three dimensional projective image space. The image space coordinates are defined as:

$$
\begin{array}{ll}
X_{1}=a \sin (\varphi / 2)-b \cos (\varphi / 2) ; & X_{3}=2 \sin (\varphi / 2) \\
X_{2}=a \cos (\varphi / 2)+b \sin (\varphi / 2) ; & X_{4}=2 \cos (\varphi / 2) .
\end{array}
$$

The mapping is injective, not bijective: there is at most one pre-image for each image point. Any image point on the real line $l$, defined by the intersection of the coordinate planes $X_{3}=X_{4}=0$, has no pre-image and therefore does not correspond to a real displacement of $E$. See Bottema and Roth, 1979, for a detailed analysis of the geometry of the image space. 
To be practical, we can remove the one parameter family of image points for coupler orientations of $\varphi=\pi$, and normalize the image space coordinates by setting $X_{4}=1$. Conceptually, this implies dividing the $X_{i}$ by $X_{4}=2 \cos (\varphi / 2)$ giving

$$
\begin{aligned}
X_{1} & =\frac{1}{2}(a \tan (\varphi / 2)-b) ; & X_{3} & =\tan (\varphi / 2) \\
X_{2} & =\frac{1}{2}(a+b \tan (\varphi / 2)) ; & X_{4} & =1 .
\end{aligned}
$$

Since each distinct displacement described by $(a, b, \varphi)$ has a corresponding unique image point, the inverse mapping can be obtained from Eqs. (3): for a given point of the image space, the displacement parameters are

$$
\begin{aligned}
\tan (\varphi / 2) & =X_{3} \\
a & =2\left(X_{1} X_{3}+X_{2}\right) /\left(X_{3}^{2}+1\right) \\
b & =2\left(X_{2} X_{3}-X_{1}\right) /\left(X_{3}^{2}+1\right)
\end{aligned}
$$

By virtue of the relationships expressed in Eqs. (3), the transformation matrix from Eq. (1) may be expressed in terms of the homogeneous coordinates of the image space. After setting $z=1$, which is done because no practical coupler will have a point at infinity, one obtains a linear transformation to express a displacement of $E$ with respect to $\Sigma$ in terms of the coordinates of the image point:

$$
\left[\begin{array}{l}
X \\
Y \\
Z
\end{array}\right]=\left[\begin{array}{ccc}
1-X_{3}^{2} & -2 X_{3} & 2\left(X_{1} X_{3}+X_{2}\right) \\
2 X_{3} & 1-X_{3}^{2} & 2\left(X_{2} X_{3}-X_{1}\right) \\
0 & 0 & X_{3}^{2}+1
\end{array}\right]\left[\begin{array}{l}
x \\
y \\
1
\end{array}\right] .
$$

\subsection{Planar Constraint Equations}

Corresponding to the kinematic constraints imposed by $R R$ - and $P R$ dyads are quadric constraint surfaces in the image space. A general equation is obtained when $(X: Y: Z)$ from Eqs. (5) are substituted into the general equation of a circle, the form of the most general constraint, Hayes, et al., 2004:

$$
K_{0}\left(X^{2}+Y^{2}\right)+2 K_{1} X Z+2 K_{2} Y Z+K_{3} Z^{2}=0 .
$$

The result is that the constraint surfaces corresponding to $R R$, and $P R$ dyads can be represented by one equation (see Hayes, et al., 2004, for how to include $R P$ - and $P P$-dyads as well). After re-arranging in terms of the constraint surface shape parameters $K_{0}, K_{1}, K_{2}, K_{3}, x$, and $y$, treating the image space coordinates $X_{1}, X_{2}$, and $X_{3}$ as constants yields:

$$
\begin{gathered}
{\left[\frac{1}{4}\left(X_{3}^{2}+1\right) x^{2}+\left(X_{2}-X_{1} X_{3}\right) x+\frac{1}{4}\left(X_{3}^{2}+1\right) y^{2}-\left(X_{1}+X_{2} X_{3}\right) y+X_{2}^{2}+X_{1}^{2}\right] K_{0}+} \\
{\left[\frac{1}{2}\left(1-X_{3}^{2}\right) x-X_{3} y+X_{1} X_{3}+X_{2}\right] K_{1}+\left[X_{3} x+\frac{1}{2}\left(1-X_{3}^{2}\right) y-X_{1}+X_{2} X_{3}\right] K_{2}+} \\
\frac{1}{4}\left(X_{3}^{2}+1\right) K_{3}=0 .
\end{gathered}
$$


For a particular dyad the associated $\left[K_{0}: K_{1}: K_{2}: K_{3}\right]$, along with the design values of the coordinates of the coupler attachment point $(x, y)$, expressed in reference frame $E$, are substituted into Eq. (7) revealing the image space constraint surface for the given dyad. The $K_{i}$ in Eqs. (6) and (7) depend on the constraints imposed by the dyad.

For $R R$-dyads $K_{0}=1$ and the surface is a hyperboloid of one sheet that intersects planes parallel to $X_{3}=0$ in circles, Hayes and Husty, 2003. The $K_{i}$ are termed circular coefficients and are defined as:

$$
\left[K_{0}: K_{1}: K_{2}: K_{3}\right]=\left[1:-X_{c}:-Y_{c}:\left(K_{1}^{2}+K_{2}^{2}-r^{2}\right)\right],
$$

where the ungrounded $R$-pair in an $R R$-dyad is constrained to move on a circle of constant radius, $r$, and fixed centre coordinates in $\Sigma,\left(X_{c}, Y_{c}\right)$.

Linear constraints result when $P R$-dyads are employed. In this case $K_{0}=0$ and the constraint surface is an hyperbolic paraboloid with one regulus ruled by skew lines that are all parallel to the plane $X_{3}=0$, Hayes and Husty, 2003. The linear coefficients are defined as

$$
\left[K_{0}: K_{1}: K_{2}: K_{3}\right]=\left[0: \frac{1}{2} L_{1}: \frac{1}{2} L_{2}: L_{3}\right],
$$

where the $L_{i}$ are line coordinates obtained by Grassmann expansion of the determinant of any two distinct points on the line, Klein, 1939. We obtain

$$
\left[K_{0}: K_{1}: K_{2}: K_{3}\right]=\left[0:-\frac{1}{2} \sin \vartheta_{\Sigma}: \frac{1}{2} \cos \vartheta_{\Sigma}: F_{X / \Sigma} \sin \vartheta_{\Sigma}-F_{Y / \Sigma} \cos \vartheta_{\Sigma}\right],
$$

where $\vartheta_{\Sigma}$ is the angle the direction of translation makes with respect to the $X$-axis, expressed in $\Sigma$ (see Figure 1), $F_{X / \Sigma}, F_{Y / \Sigma}$, represent the homogeneous coordinates $(X: Y: 1)$, expressed in reference frame $\Sigma$, of a point on the line that is fixed relative to $\Sigma$.

\section{Singular Value Decomposition}

Singular value decomposition (SVD) decomposes any given $m \times n$ matrix $\mathbf{C}$ into the product of three matrix factors (see Goulb and Van Loan, 1996, for example) such that $\mathbf{C}_{m \times n}=\mathbf{U}_{m \times m} \mathbf{S}_{m \times n} \mathbf{V}_{n \times n}^{T}$, where $\mathbf{U}$ and $\mathbf{V}$ are orthogonal $\left(\mathbf{U}^{T} \mathbf{U}=\mathbf{I}_{m}\right.$ and $\mathbf{V}^{T} \mathbf{V}=\mathbf{I}_{n}$ ), $\mathbf{S}$ is an upperdiagonal matrix whose diagonal elements are the singular values of $\mathbf{C}$ arranged in descending order (singular values are lower bounded by 0 ).

SVD explicitly constructs orthonormal bases spanning the nullspace and the range of a matrix. This has very appealing applications to any set of linear equations (or equations that may be treated as linear in the unknowns) of the form $\mathbf{C k}=\mathbf{0}$. In fact, if $\mathbf{C}_{m \times n}$ is not of full column rank, then the last $n-\operatorname{rank}(\mathbf{C})$ columns of $\mathbf{V}$ span the nullspace of $\mathbf{C}$ and any of these columns is a non-trivial solution of $\mathbf{C k}=\mathbf{0}$. For example, if $\mathbf{C}_{m \times n}$ has rank $n-1$, then the $n^{\text {th }}$ column of $\mathbf{V}$ is a solution. 


\section{Combining Type and Dimension Synthesis}

In Hayes and Zsombor-Murray, 2002, it was shown that kinematic mapping can be used for exact dimension synthesis for rigid body guidance by solving the resulting five equations generated by Eq. (7) assuming a dyad type, i.e., assigning a value to $K_{0}$. By solving the system of equations for the shape parameters $K_{1}, K_{2}, K_{3}, x$, and $y$ all in terms of $K_{0}$, one can simultaneously perform type and dimension synthesis, see Hayes and Zsombor-Murray, 2004. If $K_{0}$ is unspecified, Eq. (7) can be used to generate the homogeneous equations $\mathbf{C k}=\mathbf{0}$. The product $\mathbf{C k}$ is linear in the products of $K_{i}, x$, and $y$. Examining Eq. (7), the corresponding vector $\mathbf{k}$ is:

$$
\left[\begin{array}{llllllllllll}
K_{0} & K_{1} & K_{2} & K_{3} & K_{0} x & K_{0} y & K_{0} x^{2} & K_{0} y^{2} & K_{1} x & K_{1} y & K_{2} x & K_{2} y
\end{array}\right]^{\mathrm{T}} .
$$

It is well known that, in general, nine non-coplanar points in space define a quadric. However, if we allow $K_{0}$ to be variable (not set a priori to either 1 or 0 ), then it would appear that 6 points will determine one, possibly more, image space constraint quadrics. But, the coefficient matrix has 12 columns, suggesting that the model does not fit the physics. Hence, an exploratory experiment was devised.

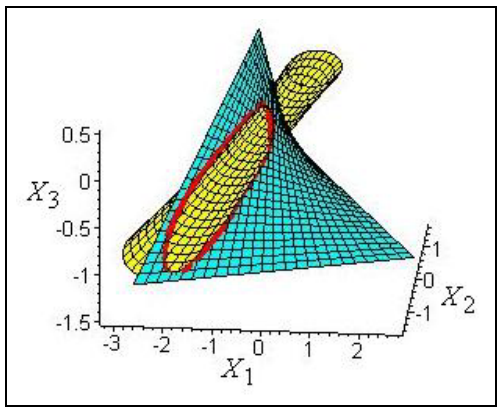

\begin{tabular}{ccc}
\multicolumn{3}{c}{ Table 1. Generating mechanism shape parameters. } \\
\hline Parameter & PR-dyad & $R R$-dyad \\
\hline$K_{0}$ & 0 & 1 \\
$K_{1}$ & -1 & -2 \\
$K_{2}$ & 1 & 0 \\
$K_{3}$ & 0 & 3 \\
$x$ & 0 & 1 \\
$y$ & 0 & 0 \\
\hline
\end{tabular}

Figure 2. Image space constraint curve.

The $P R R R$ mechanism illustrated in Figure 1 was used to generate 20 poses. The $P R$ - and $R R$-dyad parameters for this linkage are listed in Table 1, while the resulting 20 poses are listed, but only up to four decimal places, in Table 2. The curve of intersection of the constraint hyperbolic paraboloid and hyperboloid is easily parameterized and used to generate the poses, see Hayes and Husty, 2003. Figure 2 shows the intersection of the two generating surfaces. The properties of quartic image space curves of this kind are well known, and discussed in Bottema and Roth, 1979. They are the images of fourth order coupler curves. 
The coefficient matrix $\mathbf{C}$, whose elements are all functions of the image space coordinates corresponding to this set of poses is $20 \times 12$. Careful examination of Eq. (7) reveals several of the $X_{i}$ terms are identical. For example the products $K_{0} x^{2}, K_{0} y^{2}$, and $K_{3}$ all share $1 / 4\left(X_{3}^{2}+1\right)$ as a coefficient. An important outcome is that $\operatorname{rank}(\mathbf{C}) \leq 8$, here $\operatorname{rank}(\mathbf{C})=$ 6. The resulting nullspace basis vectors include linear combinations that correspond to the generating dyads. But, there is no obvious way to determine the optimal linear combinations in a way that presents a computational advantage over Cartesian-based numerical methods.

Table 2. The 20 generated poses.

\begin{tabular}{ccc}
\hline$a$ & $b$ & $\varphi$ (deg.) \\
\hline 1.0956 & 1.0956 & -5.7248 \\
1.1005 & 1.1005 & -6.0256 \\
1.1058 & 1.1058 & -6.3597 \\
1.1117 & 1.1117 & -6.7329 \\
1.1184 & 1.1184 & -7.1527 \\
1.1259 & 1.1259 & -7.6281 \\
1.1344 & 1.1344 & -8.1712 \\
1.1441 & 1.1441 & -8.7974 \\
1.1554 & 1.1554 & -9.5273 \\
1.1687 & 1.1687 & -10.3889 \\
1.1844 & 1.1844 & -11.4212 \\
1.2034 & 1.2034 & -12.6804 \\
1.2268 & 1.2268 & -14.2500 \\
1.2563 & 1.2563 & -16.2602 \\
1.2949 & 1.2949 & -18.9246 \\
1.3474 & 1.3474 & -22.6199 \\
1.4229 & 1.4229 & -28.0725 \\
1.5403 & 1.5403 & -36.8699 \\
1.7403 & 1.7403 & -53.1301 \\
2.0000 & 2.0000 & -90.0000 \\
\hline
\end{tabular}

We are looking for the two dyads that best generate the desired poses. As long as we can identify all surface shape parameters, we can add certain columns in $\mathbf{C}$ to reduce its nullity, but retain its rank. Thus we reduce the nullity of $\mathbf{C}$ to 1 , but in two different ways. Two different column combinations lead to SVD exactly identifying the generating $P R$ - and $R R$-dyads. We start with a new $20 \times 8$ coefficient matrix, $\mathcal{C}$, obtained by collecting all like coefficients in Eq. (7):

$$
\begin{array}{llll}
\mathcal{C}_{1}=X_{1}^{2}+X_{2}^{2} & \mathcal{C}_{3}=X_{2} X_{3}-X_{1} & \mathcal{C}_{5}=-\left(X_{1}+X_{2} X_{3}\right) & \mathcal{C}_{7}=\frac{1}{2}\left(1-X_{3}^{2}\right) \\
\mathcal{C}_{2}=X_{2}+\left(X_{1} X_{3}\right) & \mathcal{C}_{4}=X_{2}-\left(X_{1} X_{3}\right) & \mathcal{C}_{6}=\frac{1}{4}\left(1+X_{3}^{2}\right) & \mathcal{C}_{8}=X_{3}
\end{array}
$$

while the corresponding vector $\mathbf{k}$ becomes a new $8 \times 1$ vector $\boldsymbol{\kappa}$ :

$$
\left[\begin{array}{llllllll}
K_{0} & K_{1} & K_{2} & K_{0} x & K_{0} y & \left(K_{0}\left(x^{2}+y^{2}\right)+K_{3}\right) & \left(K_{1} x+K_{2} y\right) & \left(K_{2} x-K_{1} y\right)
\end{array}\right]^{\mathrm{T}} .
$$

The $20 \times 8$ coefficient matrix $\mathcal{C}$ can be altered by strategically combining columns yielding a $20 \times 7$ coefficient matrix which still has rank 6 . When different columns are added, the resulting nullspace vector either represents the generating $P R$-, or $R R$-dyad. In turn, the vector elements in $\boldsymbol{\kappa}$ corresponding to the added terms in Eq. (7) are transformed into a $7 \times 1$ vector, $\boldsymbol{\kappa}^{\prime}$. But, the combined $\kappa_{i}^{\prime}$ loses its utility for parameter identification.

To extract the $P R$-dyad columns 4 and 5 of $\mathcal{C}$ are added. This can be done without affecting the system because $K_{0}=0$. The $\mathbf{V}$ matrix factor 
from the SVD of the resulting coefficient matrix yields the solution. The corresponding elements of $\boldsymbol{\kappa}^{\prime}$ together with the $7^{\text {th }}$ column in $\mathbf{V}$ are listed in the first two columns of Table 3 .

The $R R$-dyad is extracted by adding columns 2 and 3 of $\mathcal{C}$. This can be done when $\left(X_{1}-X_{2} X_{3}\right) /\left(X_{1} X_{3}+X_{2}\right)$ has the same scalar value for every $X_{1}, X_{2}$, and $X_{3}$ in the data set. This happens only when the $P R$-dyad design parameters contain $K_{3}=x=y=0$. This condition makes the constraint surface curve of intersection symmetric in $X_{1}$ and $X_{2}$. An image space curve with $\operatorname{rank}(\mathcal{C})=6$, but with $P R$-dyad design parameters $K_{3} \neq x \neq y \neq 0$, can be transformed to one with symmetry in $X_{1}$ and $X_{2}$. The corresponding elements of $\boldsymbol{\kappa}^{\prime}$ together with the $7^{\text {th }}$, and the normalized $7^{\text {th }}$ column in $\mathbf{V}$ are listed in the last three columns of Table 3. The new elements of $\boldsymbol{\kappa}^{\prime}$ resulting from adding columns are indicated by $K_{45}$ and $K_{23}$, respectively.

Table 3. Nullspace vectors obtained by adding different columns of $\mathcal{C}$.

\begin{tabular}{ccccc}
\hline Column $4+5$ & Value & Column $2+3$ & Value & Value $/ K_{0}$ \\
\hline$K_{0}$ & 0 & $K_{0}$ & -0.2085 & 1 \\
$K_{1}$ & 0.7071 & $K_{23}$ & 0.2085 & -1 \\
$K_{2}$ & -0.7071 & $K_{0} x$ & -0.2085 & 1 \\
$K_{45}$ & 0 & $K_{0} y$ & 0 & 0 \\
$K_{0}\left(x^{2}+y^{2}\right)+K_{3}$ & 0 & $K_{0}\left(x^{2}+y^{2}\right)+K_{3}$ & -0.8340 & 4 \\
$K_{1} x+K_{2} y$ & 0 & $K_{1} x+K_{2} y$ & 0.4170 & -2 \\
$K_{2} x-K_{1} y$ & 0 & $K_{2} x-K_{1} y$ & 0 & 0 \\
\hline
\end{tabular}

Recall that scalar multiples of a triple of line coordinates represent one and the same line. In particular, the line coordinates for the $P R$ dyad line constraint that passes through the origin are $\lambda[-1: 1: 0]$. Note that SVD has determined that $K_{0}=0$, the remaining $K_{i}$ therefore determine a $P R$-dyad. In this case, $\lambda=-.7071$.

For the $R R$-dyad note that $K_{0} \neq 0$, indicating other than a $P R$-dyad. The elements of $\boldsymbol{\kappa}^{\prime}$ are normalized leading to the last column in Table 3. From these values we see that the Cartesian coordinates, expressed in the moving frame $E$, of the point constrained to move on a fixed circle are $(x, y)=(1,0)$. Knowing these two coordinates, and substituting them into the last two elements of $\boldsymbol{\kappa}^{\prime}$, it is seen that $K_{1}=-2$ and $K_{2}=0$, which concurs with the generating $R R$-dyad.

\section{Conclusions}

In this paper we have presented preliminary results that will be used in the development of an algorithm combining type and dimensional synthesis of planar mechanisms for $n$-pose rigid body guidance. While the 
results are preliminary, they nonetheless suggest the proposed approach of using the nullspace of the synthesis equations can form the backbone for an algorithm that combines both type and dimensional synthesis for the $n$-pose rigid body guidance problem. We can now identify generating mechanisms when $\operatorname{rank}(\mathcal{C})=5$ (PRRP's) and certain $\operatorname{rank}(\mathcal{C})=6$ (PRRR's). For general $P R R R$ 's we must transform the given image space curve into one symmetric in $X_{1}$ and $X_{2}$. We have yet to establish the conditions on the columns of $\mathcal{C}$ that will enable synthesis when $\operatorname{rank}(\mathcal{C})=7(R R R R$ 's $)$ and $\operatorname{rank}(\mathcal{C})=8$. The last case is the most interesting as it requires some form of error minimization because no planar four-bar linkage can yield $\operatorname{rank}(\mathcal{C})=8$.

\section{References}

Blaschke, W., (1911), "Euklidische Kinematik und nichteuklidische Geometrie", Zeit. Math. Phys., Vol. 60, pp. 61-91 and 203-204.

Bottema, O. and Roth, B. (1979), Theoretical Kinematics, Dover Publications, Inc. (1990), New York, N.Y., U.S.A..

Goulb, G.H. and Van Loan, C. (1996), Matrix Computations, The Johns Hopkins University Press, Baltimore, U.S.A..

Grünwald, J., (1911), "Ein Abbildungsprinzip, welches die ebene Geometrie und Kinematik mit der räumlichen Geometrie verknüpft", Sitzber. Ak. Wiss. Wien, Vol. 120, pp. 677-741.

Hayes, M.J.D. and Husty, M.L., (2003), "On the Kinematic Constraint Surfaces of General Three-Legged Planar Robot Platforms", Mechanism and Machine Theory, vol. 38, no. 5, pp. 379-394.

Hayes, M.J.D., Zsombor-Murray, P.J., and Chen, C., (2004), "Kinematic Analysis of General Planar Parallel Manipulators", ASME, Journal of Mechanical Design, in press.

Hayes, M.J.D., Zsombor-Murray, P.J., (2002), "Solving the Burmester Problem Using Kinematic Mapping", Proc. of the ASME Design Engineering Technical Conferences: Mechanisms Conference, Montréal, QC, Canada, on CD.

Hayes, M.J.D., Zsombor-Murray, P.J., (2004), "Kinematic Mapping Synthesis of Planar Linkages", Proceedings of the CSME Forum 2004, University of Western Ontario, London, ON., Canada, on CD.

Klein, F., (1939), Elementary Mathematics from an Advanced Standpoint: Geometry, Dover Publications, Inc., New York, N.Y., U.S.A..

Murray, A.P. and Pierrot, F., (1998), N-Position Synthesis of Parallel Planar RPR Platforms, Advances in Robot Kinematics: Analysis and Control, eds. Lenarčič, J., Husty, M.L., Kluwer Academic Publishers, Dordrecht, The Netherlands, pp. 69-78.

Ravani, B. and Roth, B. (1983), Motion Synthesis Using Kinematic Mappings, ASME, J. of Mechanisms, Transmissions, \& Automation in Design, Vol. 105, pp. 460-467.

Shirkhodaie, A.H. and Soni, A.H., (1987), Forward and Inverse Synthesis for a Robot with Three Degrees of Freedom, Summer Computation Simulation Conference, Montréal, Qué, Canada, pp. 851-856. 